\title{
Erratum
}

\section{Electrostatic gating of hybrid halide perovskite field-effect transistors: balanced ambipolar transport at room-temperature - ERRATUM}

\author{
Y. Meit, C. Zhangt, Z.V. Vardeny and O.D. Jurchescu
}

doi: 10.1557/mrc.2015.21, Published by Materials Research Society with Cambridge University Press, 4 May 2015.

In Mei et al. ${ }^{1}$, the authors were initially published in the incorrect order:

Y. Mei† and O.D. Jurchescu, Department of Physics, Wake Forest University, Winston-Salem, North Carolina 27109

C. Zhang† and Z.V. Vardeny, Department of Physics and Astronomy, University of Utah, Salt Lake City, Utah 84112

Correct order is, as listed:

Y. Mei†, Department of Physics, Wake Forest University, Winston-Salem, North Carolina 27109

C. Zhang †, Department of Physics and Astronomy, University of Utah, Salt Lake City, Utah 84112

Z.V. Vardeny, Department of Physics and Astronomy, University of Utah, Salt Lake City, Utah 84112

O.D. Jurchescu, Department of Physics, Wake Forest University, Winston-Salem, North Carolina 27109

The publisher regrets the mistakes. The original article has been updated with the correct order of authors.

\section{Reference}

1. Y. Mei, C. Zhang, Z.V. Vardeny, and O.D. Jurchescu: Electrostatic gating of hybrid halide perovskite field-effect transistors: balanced ambipolar transport at room-temperature. MRS Communications (2015). doi: 10.1557/mrc.2015.21.

$\dagger$ Authors contributed equally to the work. 